\title{
Stabilization of Nanoparticle Shells by Competing Interactions
}

\author{
Ethayaraja Mani, ${ }^{\dagger}$ Eduardo Sanz, ${ }^{\ddagger}$ Peter G. Bolhuis, ${ }^{\S}$ and Willem K. Kegel ${ }^{*, \dagger}$ \\ Van't Hoff Laboratory for Physical and Colloid Chemistry, Debye Institute, Utrecht University, Padualaan 8, \\ 3584 CH Utrecht, The Netherlands, School of Physics and Astronomy, James Clerk Maxwell Building, \\ The King's Buildings, University of Edinburgh, Mayfield Road, EH9 3JZ Edinburgh, United Kingdom, and \\ Van't Hoff Institute for Molecular Sciences, University of Amsterdam, Nieuwe Achtergracht 166, \\ 1018 WV Amsterdam, The Netherlands
}

Received: January 15, 2010; Revised Manuscript Received: March 9, 2010

\begin{abstract}
We demonstrate a mechanism to intrinsically stabilize a hollow shell composed of individual nanoparticles. Using Monte Carlo simulations, we show that if nanoparticles that interact via short-range attraction and long-range repulsion are assembled on a template, the resulting shell can be stabilized upon the removal of the template. The interplay of attractive and repulsive interactions provides energy barriers that dynamically arrest the particles and stabilize the shell. We present a well-defined stability region in the interaction parameters space. We find a transition from single layered to multilayered stable shell by increasing the range of attraction, and show that the mechanism is not limited to spherical shells but can also be extended to stabilize nonspherical shells such as torus shells. This study can potentially be useful in understanding and engineering the assembly of nanoparticles into hollow objects of various shapes.
\end{abstract}

\section{Introduction}

Submicrometer sized particles interacting via a combination of short-range attraction and long-range repulsion (competing interactions) exhibit rich phase behavior that is rather different from that of the particles with pure attractive interactions. ${ }^{1-5}$ Because attraction favors bulk phase separation and repulsion disfavors it, a balance between these interactions yields finite sized mesophases. ${ }^{4}$ Some of the experimentally observed mesophases are clusters in colloidal dispersions and globular protein solutions, ${ }^{1}$ stripelike and circular domains of nanoparticles in gas-liquid interfaces, ${ }^{2,3}$ gels with a locally ordered internal structure such as Bernal spirals ${ }^{9}$ or microcrystallites, ${ }^{10}$ and gels formed by a web of colloidal monolayers. ${ }^{11}$ In addition to supporting these experimental observations, several molecular simulation studies and density functional theory calculations predicted that competing interactions can also stabilize lamellar and columnar periodic structures ${ }^{6,7}$ as well as bubble phases. ${ }^{8}$

Similar modulated phases (patterns) have been observed in experiments in a wide range of other materials such as magnetic garnets, ferrofluids, amphiphilic Langmuir films, block-copolymers, and so on. ${ }^{12}$ The physical origins of some of these phases are explained by mean-field theory and lattice gas simulations using the appropriate form of competing interactions. ${ }^{12-14}$ Competing interactions are also important for the stability of some of the naturally occurring shells such as surfactant/lipid vesicles ${ }^{15}$ virus capsids ${ }^{16}$ and inorganic polyoxometalate (POM) based macroion shells, ${ }^{17}$ although in these cases the interactions are complicated because of anisotropic and patchy character of the monomers. ${ }^{18,19}$

We explore here the ability of competing interactions to stabilize another mesoscopic structure: hollow spherical shells composed of nanoparticles. Using Monte Carlo (MC) simulations, we show that if nanoparticles are engineered with short-

\footnotetext{
* To whom correspondence should be addressed. E-mail: w.k.kegel@uu.nl.

${ }^{\dagger}$ Utrecht University.

* University of Edinburgh.

${ }^{\S}$ University of Amsterdam.
}

range attraction and long-range repulsion and if they are initially assembled on a template, the resulting shell is stable against structural collapse even after the supporting template has been removed. The underlying mechanism of the stability is that competing interactions provide energy barriers that delay particle rearrangements. The stable shell essentially corresponds to a structurally trapped state that, like a gel, ages extremely slowly. As a result, the shell is kinetically stabilized. In the rest of the paper, our reference to a stable shell means a kinetically stable shell. As another example of kinetically trapped hollow structures, a two-dimensional analogue of hollow shells, namely, nanoparticle ring, was formed via evaporation induced convective self-assembly of nanoparticles on a substrate by both experiments and lattice gas simulations. ${ }^{20,21}$

There has been great interest recently in the synthesis of nanoparticle shells for prospective applications such as drug delivery vehicles, catalyst carriers, encapsulants for biomolecules, and so on. ${ }^{22} \mathrm{~A}$ template based method was reported recently, wherein an emulsion drop is used to assemble the nanoparticles in a fluid-fluid interface and subsequently the inner fluid is removed leaving a nanoparticle shell. ${ }^{23}$ To lock the particles and preserve the hollow structure intact against structural collapse, a strong short-ranged attraction is induced by treatments such as sintering, addition of oppositely charged polyelectrolytes, removal of the protective layer from the particle surface to induce van der Waals forces ${ }^{23}$ or chemical crosslinking using click chemistry on the surface of the shell. ${ }^{24}$ Our simulations results suggest that competing interactions can also preserve the shell structure. Competing interactions might be advantageous over these locking methods described above both because they permit better control over the number of layers in the shell and because the physical bonds can be made reversible (with the strength of a few $k_{\mathrm{B}} T$, with $k_{\mathrm{B}}$ being the Boltzmann constant and $T$ being the temperature). This study provides guidelines on the controlled synthesis of nanoparticle shells and sheds some light on the stability of naturally occurring shells. 


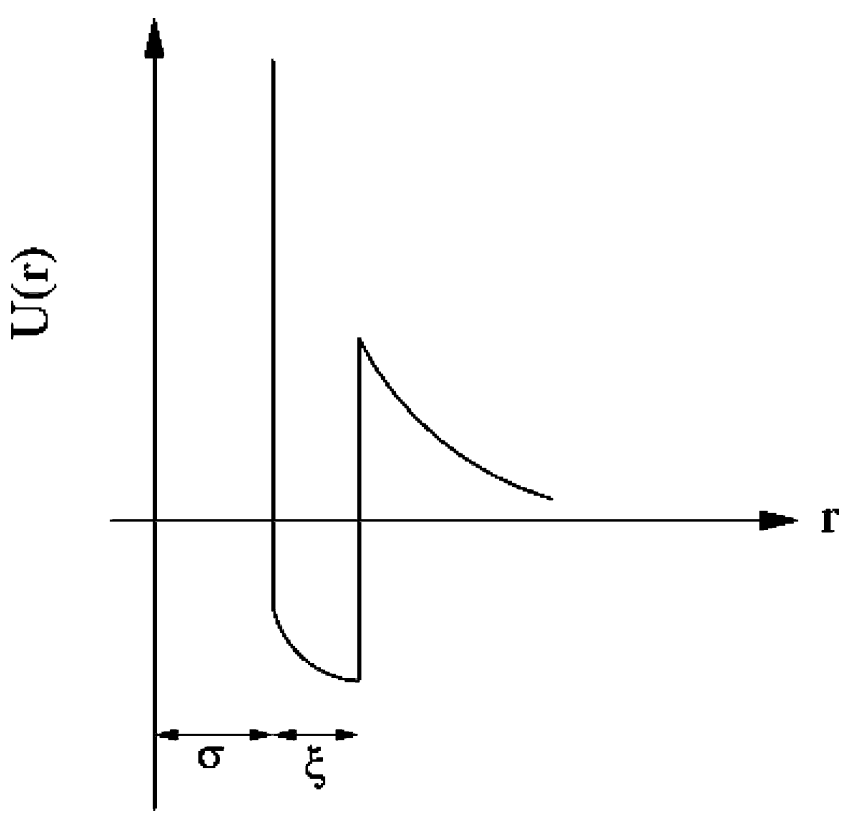

Figure 1. Sketch of the interparticle interaction potential. For $r<\sigma$, $U$ is infinite; for $\sigma \leq r<\sigma+\xi, U$ is the sum of attractive and repulsive parts; for $r \geq \sigma+\xi$, $U$ is purely repulsive. See eq 1 .

\section{Model and Simulation}

A spherically symmetric potential is chosen to represent attraction and repulsion between the particles. The attraction is represented by a square well model; the exact functional form of attraction is irrelevant if the range of attraction is smaller than the particle size. ${ }^{25} \mathrm{We}$ assume screened electrostatic interaction to represent repulsion between the particles. The total potential is given by

$$
\begin{array}{ll}
U(r)=\infty & 0 \leq r<\sigma \\
=-\varepsilon+\frac{A}{r} \mathrm{e}^{-\kappa r} & \sigma \leq r<\sigma+\xi \\
=\frac{A}{r} \mathrm{e}^{-\kappa r} & \sigma+\xi \leq r<\infty
\end{array}
$$

The particles behave as hard spheres if the interparticle separation $r<\sigma$. Here, $\sigma$ is the diameter of the particle, $\varepsilon$ is the depth of the square well (strength of attraction), $\xi$ is the width of the well (range of attraction), and $\kappa^{-1}$ is the Debye screening length. The parameter $A$ is related to the surface potential of the particle, and the exact relation can be obtained by comparing the repulsive part of eq 1 with the equation for screened electrostatic repulsion $\left(U_{\mathrm{R}}\right)$ in the Debye-Hückel approximation $\operatorname{as}^{26}$

$$
\frac{U_{\mathrm{R}}(r)}{k_{\mathrm{B}} T}=\frac{\Psi^{2} \sigma^{2} \mathrm{e}^{\kappa \sigma}}{4 \lambda_{\mathrm{B}}} \frac{\mathrm{e}^{-\kappa r}}{r}
$$

where $\Psi$ is the surface potential in units of $k_{\mathrm{B}} T$ and $\lambda_{\mathrm{B}}$ is the Bjerrum length. Therefore, the parameter $\mathrm{A}$ in eq 1 is related to $\Psi$ by the relation $A=k_{\mathrm{B}} T \Psi^{2} \sigma^{2} \mathrm{e}^{\kappa \sigma} / 4 \lambda_{\mathrm{B}}$. A sketch of the potential is given in Figure 1.

It is convenient to use reduced parameters in the simulation. Length and energy scales are expressed in units of $\sigma$ and $\varepsilon$, respectively. Reduced parameters are indicated with an overbar. Equation 1 has four independent parameters: strengths and ranges of attraction $\left(\varepsilon\right.$ and $\xi$ ) and repulsion $\left(A\right.$ and $\left.\kappa^{-1}\right)$. We fix the parameters corresponding to the attraction $\varepsilon$ (in terms of reduced temperature, $\left.\bar{T}=k_{\mathrm{B}} T / \varepsilon=0.15\right)$ and $\xi(\bar{\xi}=0.05)$ and vary the repulsive parameters, that is, $\bar{A}$ and $\bar{\kappa}^{-1}$, in a series of simulations. The repulsive interactions between the particles can be controlled in experiments by tuning $\mathrm{pH}$, concentration of salt, and composition of the solution. For example, for a given set of $\bar{A}, \bar{\kappa}^{-1}$, the required surface potential $(\Psi)$ can be calculated from the relation given above. The required electrolyte concentration $(C)$ can be calculated from the relation $C=(0.304 \bar{\kappa})$ $\sigma(n m))^{2} \cdot{ }^{26}$ In this relation, we assumed 1:1 electrolyte in aqueous medium and a temperature of $25^{\circ} \mathrm{C}$; $C$ is in the unit of molarity. Similarly, the renormalized number of charges per particle $(Q)$ can be calculated for a given $\Psi$ using the relation $Q=\Psi(2+$ $\bar{\kappa}) / 4 \bar{\lambda}_{\mathrm{B}} \cdot{ }^{26}$ Other systems interacting via competing interactions such as charged colloids grafted with DNA, charged nanoparticles forming hydrogen bonds, and charged proteins with hydrophobic patches on their surface can also be studied similarly by choosing appropriate form of attraction and repulsion in the model.

In experiments, emulsion droplets and bubbles can be used as templates to assemble particles in a close-packed layer. Particles between nanometer and micrometer in size spontaneously adsorb on the water-oil interface if $\gamma_{\mathrm{wo}}>\left|\gamma_{\mathrm{po}}-\gamma_{\mathrm{pw}}\right|$, where $\gamma_{\mathrm{wo}}, \gamma_{\mathrm{po}}$, and $\gamma_{\mathrm{pw}}$ are the interfacial energies between water-oil, particle-oil, and particle-water, respectively. ${ }^{27}$ Upon adsorbing at the interface, the particles enormously reduce the water-oil contact area $(\approx 90 \%)$, and the total energy of the system is significantly lowered. In the case of micrometer sized particles, the adsorption energy can be as high as $10^{6}$ times the thermal energy, and thus the adsorbed particle can never escape the interface. ${ }^{28}$ Compared to such a high value of adsorption energies, the interparticle interactions used in our study are negligibly small, and therefore the close-packed assembly of particles on the template is not influenced by the details of interparticle interactions. ${ }^{29}$ To mimic this adsorption process, we simply model the particles as hard sphere and generate the close-packed initial configuration for the shell.

Our MC simulations are performed in two steps. In the first step, we construct the initial shell composed of particles in close packed arrangement. We generate $N=1122$ points on the surface of a unit sphere (shell) using the construct $(\mathrm{m}, \mathrm{n})$ algorithm. ${ }^{30}$ After placing $\mathrm{N}$ particles at those points, we rescale the shell radius such that the particles (with $\sigma=1$ ) do not overlap. In such a shell, however, some of the first nearest neighbors of a given particle exist outside the chosen range of attraction $(\bar{\xi}=0.05)$. This is due to the nonuniform distribution of nearest neighbor distances in the original configuration. With little rearrangement of particles, it is possible to localize almost all the first nearest neighbors within the range of attraction and still retain the spherical shape of the shell. To achieve this, we perform MC simulation in the NPT ensemble using a hard sphere interaction between the particles, with a reduced pressure $\bar{P}=P \sigma^{3} / k_{\mathrm{B}} T=100$. The translational moves are constrained to move on the surface of the shell. This constraint and volume change moves in the NPT ensemble give a minimum radius of the shell $\left(\bar{R}_{0}=9.05\right)$, with particles in nearly close packed arrangement. We emphasize that the NPT MC simulation is performed only to obtain the initial configuration of the particles on the shell. In the second step, MC simulations are carried out to study the stability of the shell. With the initial configuration of particles on the shell obtained as above, we introduce the interparticle potential (eq 1) and examine the evolution of the shell in an NVT MC simulation in which the particles are no longer constrained to move in the surface of a sphere. 


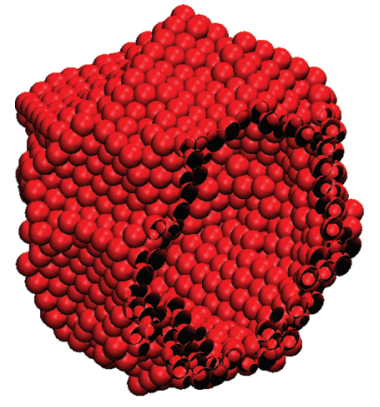

(a)

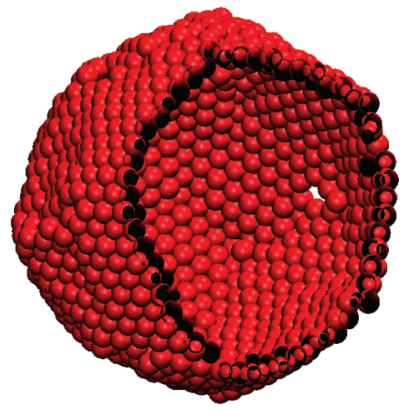

(b)

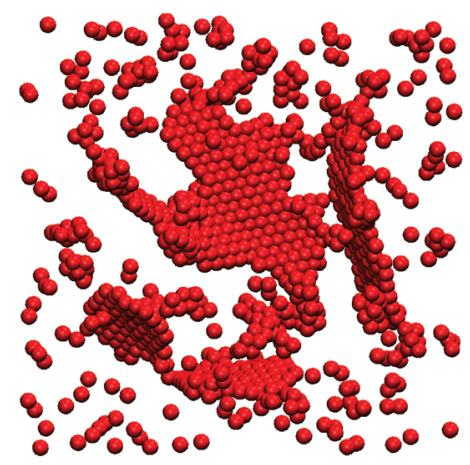

(c)

Figure 2. Snapshots of (a) collapsed $\left(\bar{A}=0.01, \bar{\kappa}^{-1}=2\right)$, (b) stable $\left(\bar{A}=0.125, \bar{\kappa}^{-1}=2\right)$, and (c) burst $\left(\bar{A}=0.6, \bar{\kappa}^{-1}=2\right)$ shells after 0.5 million MC cycles. The shells (a and b) are deliberately sliced partly to show the interiors of the collapsing and stable shells more clearly.

The length of the periodic simulation box is fixed as $4 \bar{R}_{0}$, where $\bar{R}_{0}$ is the initial radius of the shell. The cutoff $\left(\bar{r}_{\mathrm{c}}\right)$ for potential energy calculations is calculated for different sets of $\bar{A}$ and $\bar{\kappa}^{-1}$ values such that $\bar{U}\left(\bar{r}_{\mathrm{c}}\right)<0.001$ and $\bar{r}_{\mathrm{c}}$ is less than half of the box length in all our simulations. The volume fraction of particles $(1.24 \%)$ is so low that shell-shell interactions are negligible in our simulations. The MC step sizes are chosen such that the moves are accepted in the range of $40-60 \%$. The typical length of simulations is 0.5 million MC cycles, where a MC cycle consists of $N$ attempted particle moves. The stability of a shell is studied using a combination of several criteria: visual inspection of configurations, average coordination number per particle, and average distance of the particles from the center of mass. With this set of criteria, a stability region is established in the $\left(\bar{A}, \bar{\kappa}^{-1}\right)$ parameters space.

\section{Results and Discussion}

The initial shape of a shell is a perfect sphere. Depending on the relative strength of attraction and repulsion, the shell remains either stable (S), or collapses (C), or bursts (B) as the simulation proceeds. Representative snapshots of these three kinds of structures obtained from MC simulations are shown in Figure 2. Besides the visual inspection, we track the coordination number per particle $(Z)$ as a function of MC cycles to determine the status of a shell. The evolution of $Z$ for the three representative cases is shown in Figure 3a. All three shells initially have the same $Z$ value. The initial $Z$ value is slightly smaller than 6 , the limit of $2 \mathrm{D}$ close packing, because not all six neighbors are within the range of attraction with $\bar{\xi}=0.05$. For the collapsing shells, $Z$ monotonically increases with the $\mathrm{MC}$ cycles. If the repulsion is either absent or very weak,
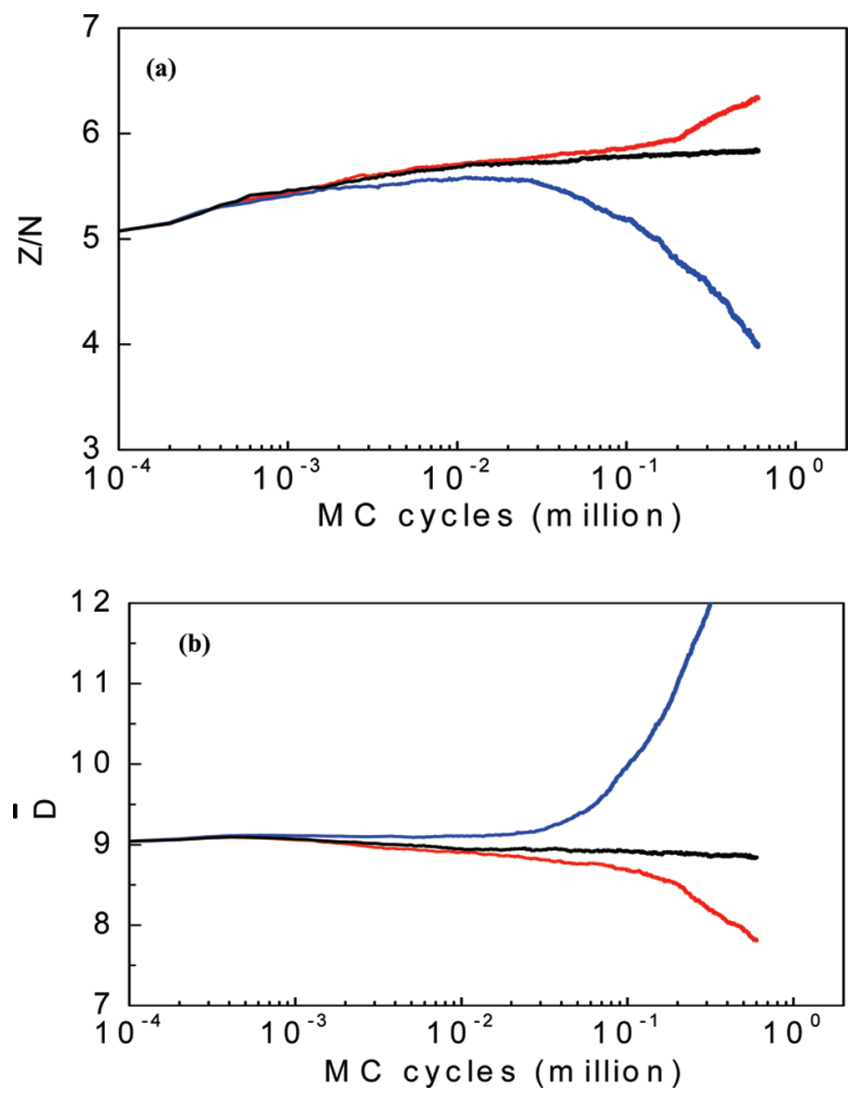

Figure 3. (a) Coordination number per particle, $Z$, and (b) average distance of the particles to the center of mass $(\bar{D})$ as a function of MC cycles. The red, black, and blue curves are for collapsing $(\bar{A}=0.01$ and $\left.\bar{\kappa}^{-1}=2\right)$, stable $\left(\bar{A}=0.125\right.$ and $\left.\bar{\kappa}^{-1}=2\right)$, and bursting $(\bar{A}=0.6$ and $\bar{\kappa}^{-1}=2$ ) shells, respectively.

particles can easily navigate within their range of attraction to find more neighbors without experiencing any significant repulsion from second nearest neighbors and beyond. Therefore, $Z$ increases beyond 6 and continues to increase further until the shell collapses into a compact three-dimensional cluster. From the snapshot shown in Figure 2a, and from the continuous increase in $Z$ shown in Figure 3a, we find that the mechanism of collapse is by local aggregation and inward buckling of the planes of particles.

For bursting shells, Figure 3 a shows that $Z$ initially increases similar to the collapsing case, but decreases progressively later. The initial configuration of the shell was generated using hard sphere interactions. On introducing the competing interactions, there are few local rearrangements of particles to increase $Z$ in all three cases. In the bursting shell, however, the strong repulsion takes over this effect and decreases $Z$. As a result of this competing effect, $Z$ shows a maximum as depicted in Figure $3 \mathrm{a}$. In the regime where $Z$ decreases, the repulsion due to the neighbors in the shell surface is so strong that the particles move away from their neighbors in order to minimize the repulsion. Visual inspections of the transient stages reveal that initially a few particles escape from the surface leaving some holes behind. Subsequently, more particles escape from the rims of these holes, which is facilitated by the fact that rim particles have to break less than 6 bonds to escape. This mechanism finally leads to bursting of the shell into small planar objects as shown in Figure 2c.

To obtain a stable shell, the competing interactions should produce an energy barrier for the local rearrangement of individual particles. If such a condition is met, the shell remains 


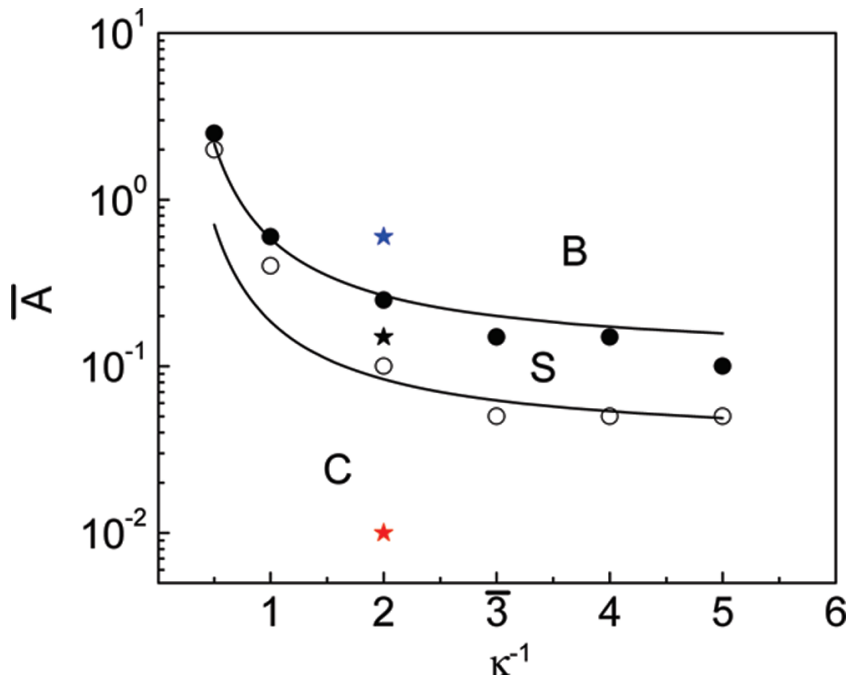

Figure 4. Stability diagram. C, S, and B denote collapsing, stable, and bursting shells, respectively. The loci of filled circles and open circles denote the boundaries obtained from simulation, while the curves are calculated from a scaling approach (see text). The red, black, and blue stars are the representative state points corresponding to, respectively, collapsing, stable, and bursting shells as shown in Figure 2.

stable, and $Z$ for a stable shell is nearly constant $(\sim 6)$, varying extremely slowly as shown in Figure 3a. Such a slow aging is characteristic of trapped structures like gels. ${ }^{31}$ As the rearrangements of particles become highly unlikely due to the large activation barrier, the stability of the shell is kinetic rather than thermodynamic. In some stable shells, a degree of faceting appears evident as a consequence of small local rearrangements of particles that minimize the overall energy (see Figure 2b). A similar faceting was observed by Abkarian et al. ${ }^{32}$ in their experiments on Pickering bubbles as the inner fluid is evaporated slowly. Nevertheless, the origin of faceting observed in their experiments was due to a balance between capillary forces of the inner fluid and electrostatic repulsion between the particles, whereas for our nanoparticle shell it is purely due to interparticle interactions, in particular by the repulsive interaction, that is minimized by locally flattening the shell. In addition to $Z$, the average distance of the particles to the center of mass $(\bar{D})$ also indicates the state of the shell, as shown in Figure $3 \mathrm{~b}$. We identify collapsing, stable, and bursting shells if $\bar{D}<\bar{R}, \bar{D} \approx \bar{R}$ (within a few percentage), and $\bar{D}>\bar{R}$, respectively. With the combinations of these three criteria, a series of simulations on a set of $\bar{A}, \bar{\kappa}^{-1}$ are performed and the shells are classified accordingly.

We find from the simulation results a narrow stability region in the parameters space of $\bar{A}$ and $\bar{\kappa}^{-1}$, shown in Figure 4 . The top boundary (locus of the filled circles) and the bottom boundary (locus of the open circles) correspond to the onset of burst and collapse, respectively, and within these two boundaries lies the stable region. Figure 4 validates our choice of competing interactions, as there is a wide range of values of repulsive parameters $\left(\bar{A}\right.$ and $\left.\bar{\kappa}^{-1}\right)$ that can stabilize shells. The stability region presented here can provide guidance for the synthesis of shells by engineering colloids or nanoparticles with tuned interactions. In addition, it can potentially shed light in the understanding of naturally occurring shells such as virus capsids ${ }^{13}$ or POM shells. ${ }^{14}$ Although the interactions between the constituents of these systems are certainly more complex than the spherically symmetric potential we study here, it is useful to know the minimum requirement for stability of these intriguing molecular assemblies before entering into more subtle details to describe their interactions.
We also performed simulations with shells of $N=272\left(\bar{R}_{0}\right.$ $=4.49)$ and $432\left(\bar{R}_{0}=5.64\right)$ particles. We found that the stability region for these shells spanned almost the same range as that for the shell of 1122 particles, as given in Figure 4. Nevertheless, in a very large shell, other types of interactions such as gravitational field may play a role and our mechanism may (or may not) apply in those size regimes. As trapped structures, the stable shells slowly age into a lower free energy state, and it is important to recognize that the stability boundaries of Figure 4 may slowly vary with time, narrowing the stability region slightly. Nevertheless, the aging is extremely slow as shown in Figure 3 b. A fit to the simulation data shows that $\bar{D}$ decays logarithmically with time (see the Supporting Information). If the hollow shell formed by 1122 particles were to collapse to a compact spherical cluster as the final state, the radius of the cluster would be $5.2 \sigma$. Using the fitted equation, we can estimate the time required for this transformation, $\bar{t}=$ $\exp (136) \approx 10^{59}$, where time $\bar{t}$ is nondimensionalized by Brownian time $(\tau)$, which is defined as the time taken for the particle to diffuse over its own diameter. Using the Einstein-Stokes equation for diffusivity, $\tau$ for a $10 \mathrm{~nm}$ particle is $\sim 10 \mu \mathrm{s}$ in water at room temperature. The aging time scale is then $\approx 10^{54}$ s. This is an infinitely long time scale if the shell ages purely by diffusion. Nevertheless, we note that the aging mechanism may be more complicated than by pure diffusion because it may involve a cooperative effect of more than one particle. Therefore, it should be possible to experimentally detect shells within the stability region and to use them for practical purposes during their lifetime.

We now put forward a simple scaling approach to explain the boundaries of the stability region in Figure 4. In an ideal stable shell, initially particles are close packed and localized in the square well. For a shell to burst, each particle has to escape from the attractive well of its neighbors. The total energy, the sum of attraction and repulsion, of a particle in the shell is then given by $\bar{U}_{\mathrm{B}}=-6+\bar{A} \sum_{i} n_{i} \mathrm{e}^{-\overline{\kappa_{i}} / \bar{r}_{i}}$, where the summation is over circles of the $n_{i}$ neighbors at a distance $\bar{r}_{i}$. The escape rate is related to an energy barrier given by $-\bar{U}_{\mathrm{B}}$. To estimate the barrier, we calculated $n_{i}$ and $\bar{r}_{i}$ for the first five circles of neighbors in a hexagonal packing of a plane in the limit of infinite size of a shell. This yielded $n_{i}=\{6,6,6,12,6\}$ and $\bar{r}_{i}$ $=\left\{\bar{r}_{\mathrm{e}}, \sqrt{ } 3 \bar{r}_{\mathrm{e}}, 2 \bar{r}_{\mathrm{e}}, \sqrt{ } 7 \bar{r}_{\mathrm{e}}, 3 \bar{r}_{\mathrm{e}}\right\}$, where $\bar{r}_{\mathrm{e}}(\approx 1+\bar{\xi} / 2=1.025)$ is the equilibrium distance between the particles at close packing. We solved the equation above for $\bar{A}$ as a function of $\bar{\kappa}^{-1}$ and fitted it to the data obtained from simulation corresponding to the onset of bursting with $\bar{U}_{\mathrm{B}}$ as the only free parameter. The best fit is obtained for $\bar{U}_{\mathrm{B}}=-4.0$.

Similarly, for a collapsing shell, a particle has to overcome the repulsion from all of its neighbors before it reaches the attractive well of its new neighbors. The energy barrier is then due to repulsion only, that is, $\bar{U}_{\mathrm{C}}=\bar{A} \Sigma_{i} n_{i} \mathrm{e}^{-\bar{\kappa} \bar{r}_{i} / \bar{r}_{i}}$. We fit the boundary corresponding to collapse (see Figure 4 ) with this equation using $\bar{U}_{\mathrm{C}}=0.6$. The simulations were performed with a reduced temperature $\bar{T}=0.15$, which corresponds to $\varepsilon=$ $6.7 k_{\mathrm{B}} T$. Therefore, the energy barriers for bursting and collapse in dimensional units correspond to $26 k_{\mathrm{B}} T$ and $4 k_{\mathrm{B}} T$, respectively. It is interesting to note that a stable shell in the middle of the stability region is more likely to collapse at infinite time because the energy barrier for collapsing is smaller than that for bursting. These calculations demonstrate that a shell corresponding to the stable region has to overcome a substantial energy barrier in order to either burst or collapse and, therefore, remains in a trapped state. While the attraction strength contributes mostly 
TABLE 1: Optimum Values of Surface Potential ( $\Psi$ ), Concentration of Electrolyte $(C)$, and Charges $(Q)$ Corresponding to the Stable Region of the Stability Diagram for Two Different Particle Diameters: 2.5 and $100 \mathbf{n m}^{a}$

\begin{tabular}{|c|c|c|c|c|c|c|c|}
\hline \multirow[b]{2}{*}{$\bar{\kappa}$} & \multirow[b]{2}{*}{$\bar{A}$} & \multicolumn{3}{|c|}{$\sigma=2.5 \mathrm{~nm}$} & \multicolumn{3}{|c|}{$\sigma=100 \mathrm{~nm}$} \\
\hline & & $\Psi\left(k_{\mathrm{B}} T\right)$ & $C(\mathrm{mM})$ & $Q$ & $\Psi\left(k_{\mathrm{B}} T\right)$ & $C(\mu \mathrm{M})$ & $Q$ \\
\hline 2 & 2.5 & 1.58 & 59.1 & 5.6 & 0.25 & 36.96 & 35.7 \\
\hline 1 & 0.6 & 1.27 & 14.7 & 3.4 & 0.2 & 9.24 & 21.6 \\
\hline 0.5 & 0.25 & 1.06 & 4.0 & 2.4 & 0.17 & 2.3 & 15 \\
\hline
\end{tabular}

${ }^{a}$ Parameters are calculated for an aqueous solution.

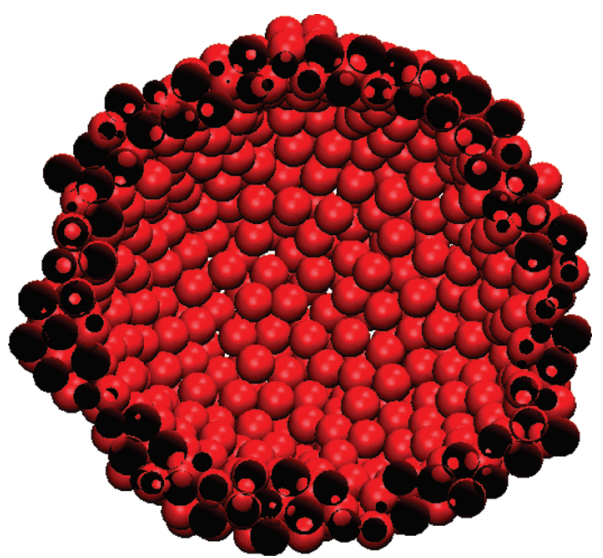

Figure 5. Section of double-layered shell obtained from MC simulation with interaction parameter $\bar{A}=0.125, \bar{\kappa}^{-1}=2$, and $\bar{\xi}=0.3$, after 2.5 million MC cycles.

to the energy barrier for bursting, the long-range repulsion contributes to the energy barrier for collapsing.

We estimate the physical variables corresponding to the stable region and verify whether they can be tuned in experiments. From the set of $\bar{A}, \bar{\kappa}^{-1}$ corresponding to the onset of burst in Figure 4 , we calculate $\Psi, C$, and $Z$ for an aqueous solution. These variables are calculated for two representative particle diameters $(\sigma)$ of 2.5 and $100 \mathrm{~nm}$, and are given in Table 1. For $\sigma=2.5 \mathrm{~nm}$, the optimum surface potential ranges from $1 k_{\mathrm{B}} T$ to $1.5 k_{\mathrm{B}} T$, the charges per particle range from 2 to 6 , and the optimum salt concentration ranges from 4 to $60 \mathrm{mM}$. Several experiments have shown evidence for such highly charged nanoparticles. Verhoeff et al. ${ }^{33}$ reported a surface potential of $-2.8 k_{\mathrm{B}} T(-70 \mathrm{mV})$ for $2.5 \mathrm{~nm}$ sized $\mathrm{POM}$ in aqueous solution. For $\sigma=2.5 \mathrm{~nm}$, using $\kappa^{-1}=680 \mathrm{~nm}$ (in water at $\mathrm{pH}=7$ ) and $\lambda_{\mathrm{B}}=0.7 \mathrm{~nm}$ (in water) at room temperature, from the relation given in the Model and Simulation section, we get -5 charges per POM.
Nanoparticles of other materials may acquire charges either by preferential adsorption of charged species (ionic surfactants, capping agents, etc) or by dissociation of surface groups (e.g., silica particles acquire charges by dissociating their silanol groups). For example, Reincke et al. ${ }^{34}$ have reported a surface potential of $-2 k_{\mathrm{B}} T(\approx-50 \mathrm{mV})$ for $3 \mathrm{~nm}$ gold nanoparticles capped with mercaptopropionic acid, giving rise to -4 charges per particle.

For $\sigma=100 \mathrm{~nm}$, the optimum surface potential ranges from 0.17 to $0.25 k_{\mathrm{B}} T$, the charges per particle range from 15 to 35 , and the optimum salt concentration ranges from 2 to $37 \mu \mathrm{M}$. These ranges of values for $\Psi, C$, and $Z$ can be readily achieved either by engineering the surface of the particles or by varying conditions of the solvent. We note that, for $\sigma>100 \mathrm{~nm}$, the optimum salt concentration falls much below $\sim 1 \mu \mathrm{M}$, and in such cases it may be appropriate to prepare shells in apolar solvents, because in aqueous solutions the lowest limit is $0.1 \mu \mathrm{M}$. Thus, the simulation and an estimate of $\Psi, C$, and $Z$ together establish a demarcation on the formation of stable shells in polar and apolar solvents. We note that the stability region shown in Figure 4 is given for $\bar{T}=0.15$ (an attractive energy per contact of $6.7 k_{\mathrm{B}} T$ ) and $\bar{\xi}=0.05$, and any variations in these two parameters will influence the stability region and the corresponding physical variables.

To study the sensitivity of the range of attraction $(\bar{\xi})$ on the stability region, we performed simulations with $\bar{\xi}=0.1,0.2$, and 0.3 . We find that, for $\bar{\xi} \leq 0.2$, single layered shells are stable within the stability region. However, a new feature emerges for $\bar{\xi}=0.3$ and beyond: the single layered shell shrinks until it forms a double layered shell of particles that remains stable afterward. A sectional view of such a double layered shell is shown in Figure 5. In such a configuration, each particle has neighbors in the third dimension as well. Though the initial template has only a single layer of particles, by increasing $\bar{\xi}$, it is possible to synthesize multilayered shells.

The role of the template is crucial in stabilizing a shell. We carried out MC simulation with parameters corresponding to a stable shell $\left(\bar{A}=0.125, \bar{\kappa}^{-1}=2\right)$ but with a random initial configuration, and observed that the particles aggregated locally to form a cluster-fluid. Although the cluster-fluid and the shell have almost equal potential energies, the former is entropically favored over the latter. Therefore, a shell will not form spontaneously, but if particles are initially assembled on a template, the shell can be stabilized by competing interactions. A recent study showed that a template can also be provided temporarily by a reaction mechanism for the self-assembly of wheel-shaped polyoxometalates. ${ }^{35}$
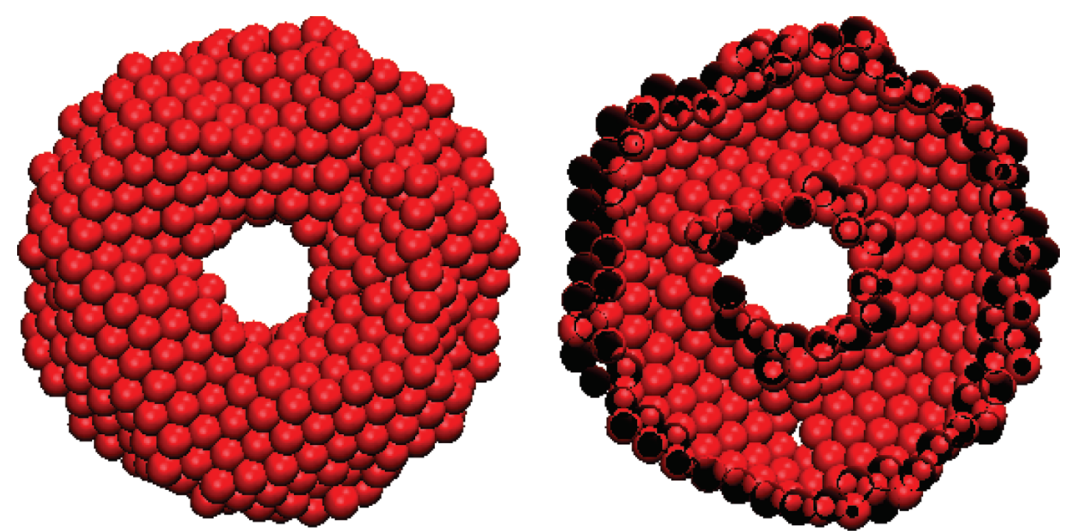

Figure 6. Snapshots of a stable torus shell (left) and a sectional view (right). Simulation is performed with $\bar{A}=0.1, \bar{\kappa}^{-1}=2$ and for 0.5 million MC cycles. 
The mechanism is not limited only to spherical shells but can be extended, in principle, to stabilize nonspherical shells as well. Experimental methods are now available to synthesize nonspherical templates such as particle stabilized ellipsoidal, toroidal bubbles and foams using a phenomenon called interfacial jamming. ${ }^{36}$ In this mechanism, two particle-covered spherical droplets (or bubbles) are fused together by squeezing them between two glass plates. The fused droplet is unable to relax to spherical shape by expelling excess particles because of very high adsorption energies. Therefore, the particles are jammed at the interface and stabilize the ellipsoidal droplet. Similarly, the toroid shaped bubble is stabilized by introducing a hole in the bubble and allowing the particle to jam at the interface. $^{36}$

To illustrate the extension of the mechanism, we performed simulations using a torus shaped template with 500 particles as an example. The initial configuration of the torus is obtained in the same way as the spherical shell described previously. As expected, we find a stable, single layered torus shell being stabilized by competing interactions, as shown in Figure 6. Our study thus opens up a new possibility in stabilizing any arbitrary shaped and closed shells using simple spherically symmetric interactions. As long as the individual particles interactions can be tuned, they can be templated in a variety of shapes and stabilized for various applications.

To test this mechanism experimentally, the key challenge would be the removal of the template. If an emulsion is used as template, the inner fluid can be removed either by evaporation or by solubilization using a common solvent. In the solubilization approach, the oil-water interface disappears altogether as the inner fluid is solubilized in a common solvent. As a result, the shell is exposed to a homogeneous fluid inside and outside. The stability diagram then suggests whether such a shell is stable or unstable to collapse/burst. Because the evaporation route induces a strong capillary force that organizes the particles into a compact cluster irrespective of the interparticle interactions, ${ }^{37}$ the solubilization approach is preferred to test this mechanism, as in this case capillary forces are absent.

\section{Conclusion}

Spherically symmetric competing interactions (a combination of short-range attraction and long-range repulsion) have so far explained the formation of a variety of mesophases and colloidal gels. Using Monte Carlo simulations, we have showed in this Article that competing interactions can take part in the stabilization of hollow structures composed of nanoparticles. We have showed that particles interacting via competing interactions can form a stable shell if they are initially assembled over a template. Upon removal of the template, the particles are trapped on the surface of the shell, rendering the structure kinetically stable. The stable shell ages very slowly, in an analogous way to colloidal gels. Stable shells are observed within a well-defined region in the interaction parameters space. We report a stability diagram providing optimum values for the potential parameters. Values of the physical variables corresponding to the stability region are experimentally achievable, so the mechanism put forward here can be readily tested in experiments. A simple scaling analysis is presented to calculate the associated energy barrier to be overcome by the particles to collapse or burst the shell. From this analysis, we explain the boundaries of the stability region reasonably well. We also showed a transition from a single layered shell to a multilayered shell upon increasing the attraction range. The generality of the mechanism in stabilizing any closed shape is demonstrated by stabilizing a single layered hollow torus shell using competing interactions.

Acknowledgment. This work was funded by a VICI grant from NWO.

Supporting Information Available: Estimation of aging time scale for nanoparticle shells. This material is available free of charge via the Internet at http://pubs.acs.org.

\section{References and Notes}

(1) Stradner, A.; Sedgwick, H.; Cardinaux, F.; Poon, W. C. K.; Egelhaaf, S. U.; Schurtenberger, P. Nature 2004, 432, 492-495.

(2) Gilbert, B.; Lu, G.; Kim, C. S. J. Colloid Interface Sci. 2007, 313, $152-159$.

(3) Ruiz-Garcia, J.; Gamez-Corrales, R.; Ivlev, B. I. Phys. Rev. E 1998, $58,660-663$.

(4) Groenewold, J.; Kegel, W. K. J. Phys. Chem. B 2001, 105, 1170211709 .

(5) Sear, R. P.; Chung, S. W.; Markovich, G.; Gelbart, W. M.; Heath, J. R. Phys. Rev. E 1999, 59, R6255-R6258.

(6) de Candia, A.; Gado, E. D.; Fierro, A.; Sator, N.; Tarzia, M.; Coniglio, A. Phys. Rev. E 2006, 74, $0104031-0104034$.

(7) Li, Z.; Wu, J. J. Chem. Phys. 2009, 130, 1651021-1651026.

(8) Archer, A. Phys. Rev. E 2008, 78, 0314021-03140210.

(9) Campbell, A. I.; Anderson, V. J.; van Duijneveldt, J. S.; Bartlett, P. Phys. Rev. Lett. 2005, 94, 2083011-2083014.

(10) Zhang, T. H.; Groenewold, J.; Kegel, W. K. Phys. Chem. Chem. Phys. 2009, 11, 10827-10830.

(11) Sanz, E.; White, K. A.; Clegg, P. S.; Cates, M. E. Phys. Rev. Lett. 2009, 103, 2555021-2555024.

(12) Seul, M.; Andelman, D. Science 1995, 267, 476-483.

(13) Chen, L. Q.; Khachaturyan, A. G. Phys. Rev. Lett. 1993, 70, 14771480.

(14) Sagui, C.; Desai, R. C. Phys. Rev. Lett. 1993, 71, 3995-3998.

(15) Kaler, E. W.; Murthy, A. K.; Rodriguez, B. E.; Zasadzinski, J. A. N. Science 1989, 245, 1371-1374.

(16) Baker, T. S.; Olson, N. H.; Fuller, S. D. Microbiol. Mol. Biol. Rev. 1999, 63, 862-922.

(17) Liu, T. J. Am. Chem. Soc. 2002, 124, 10942-10943.

(18) Drouffe, J.-M.; Maggs, A. C.; Leibler, S. Science 1991, 254, $1353-$ 1356.

(19) Kegel, W. K.; van der Schoot, P. Biophys. J. 2004, 86, 3905-3913.

(20) Yosef, G.; Rabani, E. J. Phys. Chem. B 2006, 110, 20965-20972.

(21) Vancea, I.; Thiele, U.; Pauliac-Vaujour, E.; Stannard, A.; Martin, C. P.; Blunt, M. O.; Moriarty, P. J. Phys. Rev. E. 2008, 78, 04160110416015 . 4019 .

(22) Lou, X. W.; Archer, L. A.; Yang, Z. Adv. Mater. 2008, 20, 3987-

(23) Dinsmore, A. D.; Hsu, M. F.; Nikolaides, M. G.; Marquez, M.; Bausch, A. R.; Weitz, D. A. Science 2002, 298, 1006-1009.

(24) Samanta, B.; Patra, D.; Subramani, C.; Ofir, Y.; Yesilbag, G.; Sanyal, A.; Rotello, V. M. Small 2009, 5, 685-688.

(25) Lu, P. J.; Zaccarelli, E.; Ciulla, F.; Schofield, A. B.; Sciortino, F.; Weitz, D. A. Nature 2008, 453, 499-504.

(26) Verwey, E. J. W.; Overbeek, J. T. G. Theory of Stability of Lyophobic Colloids; Dover Publications: Mineola, NY, 1999.

(27) Kralchevsky, P. A.; Nagayama, K. Adv. Colloid Interface Sci. 2000, $85,145-192$.

(28) Pieranski, P. Phys. Rev. Lett. 1980, 45, 569-572.

(29) Unlike the screened Coulombic repulsion, if long-range dipolar repulsion operates between the particles, then the particles may have to jump over the repulsive barrier to reach a minimum. For example, Nikolaides et al. showed that $1.5 \mu \mathrm{m}$ sized PMMA particles assembled on a waterin-oil droplet in hexagonal arrangement, but the interparticle distance was reported to be $5.7 \mu \mathrm{m} .{ }^{38}$ In such a scenario, close-packing (an arrangement, where particles lie within the attractive well of the neighbors) can be achieved by using capillary interactions. By slowly evaporating the inner fluid, the total surface area of the droplet (or bubble) can be reduced while keeping the number of adsorbed particles per drop constant. Then the attractive capillary forces would organize the particles in close-packed structure. This methodology was used in the experiments reported by Abkarian et al. ${ }^{32}$ Clegg et al. reported a different approach in assembling particles on emulsion droplets via fluid demixing. ${ }^{39}$ In this approach, a mixture of two liquids containing colloidal particles is cooled to a temperature where the two liquids demix. Because demixing occurs via nucleation of a new phase, the particles readily adsorb as a close-packed 
monolayer on the nucleated drops. Another possibility would be to use microfluidic channels such as in the case of Subramanian et al., where convective flow of particles can overcome the repulsive barrier. ${ }^{40}$

(30) http://phy.syr.edu/condensedmatter/thomson/thomsonapplet.htm.

(31) Zaccarelli, E. J. Phys.: Condens. Matter 2007, 19, 323101.

(32) Abkarian, M.; Subramanian, A. B.; Kim, S. H.; Larsen, R. G.; Yang, S. M.; Stone, H. A. Phys. Rev. Lett. 2007, 99, 1883011-1883014.

(33) Verhoeff, A. A.; Kistler, M. L.; Bhatt, A.; Pigga, J.; Groenewold, J.; Klokkenburg, M.; Veen, S.; Roy, S.; Liu, T.; Kegel, W. K. Phys. Rev. Lett. 2007, 99, 0661041-0661044.

(34) Reincke, F.; Kegel, W. K.; Zhang, H.; Nolte, M.; Wang, D.; Vanmaekelbergh, D.; Mohwald, H. Phys. Chem. Chem. Phys. 2006, 8, 3828-3835.

(35) Miras, H. N.; Cooper, G. J. T.; Long, D.; Bogge, H.; Muller, A.; Streb, C.; Cronin, L. Science 2010, 327, 72-74.
(36) Subramanian, A. B.; Abkarian, M.; Mahadevan, L.; Stone, H. A. Nature 2005, 438, 930.

(37) Lauga, E.; Brenner, M. P. Phys. Rev. Lett. 2004, 93, 23830112383014 .

(38) Nikolaides, M. G.; Bausch, A. R.; Hsu, M. F.; Dinsmore, A. D.; Brenner, M. P.; Gay, C.; Weitz, D. A. Nature 2002, 420, 299-301.

(39) Clegg, P. S.; Herzig, E. M.; Schofield, A. B.; Horozov, T. S.; Binks, B. P.; Cates, M. E.; Poon, W. C. K. J. Phys.: Condens. Matter 2005, 17 , S3433-3438.

(40) Subramanian, A. B.; Abkarian, M.; Stone, H. A. Nat. Mater. 2005, 4, 553-556.

JP1004067 